\title{
On a Multiple Hilbert Type Integral Inequality with the Homogeneous Kernel of 0-Degree
}

\author{
Baoju Sun \\ Department of Mathematics \\ Zhejiang University of Water Resources and Electric Power, Hangzhou, Zhejiang 310018, China \\ sunbj@zjweu.edu.cn
}

Keywords: Hilbert inequality; multiple Hilbert type inequality; best possible constant Abstract. By introducing a homogeneous kernel of 0-degree with an independent parameter $\lambda$, and estimating the weight function through the real function techniques, we give a multiple Hilbert type integral inequality with a best possible constant factor, in which the best possible constant factor also contains hypergemetric function.

\section{Introduction}

The well known Hilbert's integral inequality is given by (see $[2,8])$

$$
\int_{0}^{\infty} \int_{0}^{\infty} \frac{f(x) g(y)}{x+y} \mathrm{~d} x \mathrm{~d} y<\frac{\pi}{\sin (\pi / p)}\left[\int_{0}^{\infty} f^{p}(t) \mathrm{d} t\right]^{1 / p}\left[\int_{0}^{\infty} g^{q}(t) \mathrm{d} t\right]^{1 / q},
$$

where the constant factor $\pi / \sin (\pi / p)$ is the best possible. Hilbert's integral inequality is important in analysis and applications. During the past few years, many researchers obtained various generalizations, variants and extensions of the inequality of (1) (see [1, 6, 7, 9-14] and the references cited therein).

At present, because of the requirement of higher-dimensional harmonic analysis and higher-dimensional operator theory, multiple Hilbert's type integral inequalities have been studied. Y. Hong, B Yang and J Kuang etc. obtained some multiple Hilbert's type integral inequalities (see $[3,4,7,13,14])$.

The main objective of this paper is to build a multiple Hilbert type integral inequalities with the homogeneous kernel of 0 -degree. For this reason, we introduce signs as

$\square_{n}^{+}=\left\{x=\left(x_{1}, x_{2}, \mathrm{~L}, x_{n}\right): x_{1}, x_{2}, \mathrm{~L}, x_{n}>0\right\},\|x\|=\left(x_{1}^{2}+x_{2}^{2}+\mathrm{L}+x_{n}^{2}\right)^{\frac{1}{2}}$, and we agree on $\|x\|<c$ representing $\left\{x \in \square_{n}^{+}:\|x\|<c\right\}$.

\section{Lemmas}

We state the Hypergeometric function $F(\alpha, \beta, \gamma, z)$ as follows[15]:

If $\operatorname{Re}(\gamma)>\operatorname{Re}(\beta)>0,|\arg (1-z)|<\pi$, then

$$
F(\alpha, \beta, \gamma, z)=\frac{\Gamma(\gamma)}{\Gamma(\beta) \Gamma(\gamma-\beta)} \int_{0}^{1} t^{\beta-1}(1-t)^{\gamma-\beta-1}(1-z t)^{-\alpha} \mathrm{d} t
$$

Where $\Gamma(\alpha)=\int_{0}^{\infty} x^{\alpha=1} e^{-x} \mathrm{~d} x$. Let $z=-1, \beta=\alpha>0, \gamma=\alpha+1$, we have

$$
\int_{0}^{1} t^{\alpha-1}(1-t)^{-\alpha} \mathrm{d} t=\frac{1}{\alpha} F(\alpha, \alpha, 1+\alpha,-1)
$$

Lemma 1 ([14]) If $f(\tau)$ is a measurable function, then 


$$
\begin{aligned}
& \int_{x_{1}, x_{2}, \mathrm{~L}, x_{n}>0 ; x_{1}^{2}+x_{2}^{2}+\mathrm{L}+x_{n}^{2} \leq 1} f\left(x_{1}^{2}+x_{2}^{2}+\mathrm{L}+x_{n}^{2}\right) d x_{1} d x_{2} \mathrm{~L} d x_{n}=\frac{\Gamma^{n}\left(\frac{1}{2}\right)}{2^{n} \Gamma\left(\frac{n}{2}\right)} \int_{0}^{1} f(\tau) \tau^{\frac{n}{2}-1} d \tau, \\
& \int_{x_{1}, x_{2}, \mathrm{~L}, x_{n}>0 ; x_{1}^{2}+x_{2}^{2}+\mathrm{L}+x_{n}^{2} \geq 1} f\left(x_{1}^{2}+x_{2}^{2}+\mathrm{L}+x_{n}^{2}\right) d x_{1} d x_{2} \mathrm{~L} d x_{n}=\frac{\Gamma^{n}\left(\frac{1}{2}\right)}{2^{n} \Gamma\left(\frac{n}{2}\right)} \int_{1}^{\infty} f(\tau) \tau^{\frac{n}{2}-1} d \tau,
\end{aligned}
$$

Lemma 2 If $p>1,1 / p+1 / q=1, n \in \square, 0<\lambda<1$, Define the weight function $w_{\lambda}(x)$ as: $w_{\lambda}(x)=\int_{\square_{+}^{n}}\left(\frac{\min \{\|x\|,\|y\|\}}{\|x\|+\|y\|}\right)^{\lambda}\|y\|^{-n} \mathrm{~d} y$. Then $w_{\lambda}(x)=\frac{\Gamma^{n}\left(\frac{1}{2}\right)}{2^{n-1} \Gamma\left(\frac{n}{2}\right)} C(\lambda)=H_{n}(\lambda)$. Where $C(\lambda)=\frac{2}{\lambda} F(\lambda, \lambda, 1+\lambda,-1)$

Proof. By using Lemma 1, $w_{\lambda}(x)=\frac{\Gamma^{n}\left(\frac{1}{2}\right)}{2^{n} \Gamma\left(\frac{n}{2}\right)} \int_{0}^{\infty}\left(\frac{\min \left(1, \frac{\sqrt{t}}{\|x\|}\right)}{1+\frac{\sqrt{t}}{\|x\|}}\right)^{\frac{-n}{2}} t^{\frac{n}{2}-1} \mathrm{~d} t$ $=\frac{\Gamma^{n}\left(\frac{1}{2}\right)}{2^{n} \Gamma\left(\frac{n}{2}\right)} 2 \int_{0}^{\infty}\left(\frac{\min (1, u)}{1+u}\right)^{\lambda} \frac{1}{u} \mathrm{~d} u=\frac{\Gamma^{n}\left(\frac{1}{2}\right)}{2^{n-1} \Gamma\left(\frac{n}{2}\right)} C(\lambda)$.

. The lemma 2 is proved.

Lemma 3 If $p>1,1 / p+1 / q=1, n \in \square, 0<\lambda<1$, $0<\varepsilon<\lambda /(2 q)$, then

$$
A:=\int_{\|x\|_{\alpha} \geq 1} \int_{\|y\|_{\alpha} \geq 1}\left(\frac{\min \{\|x\|,\|y\|\}}{\|x\|+\|y\|}\right)^{\lambda}\|x\|^{-n-\frac{\varepsilon}{p}}\|y\|^{-n-\frac{\varepsilon}{q}} \mathrm{~d} x \mathrm{~d} y \geq\left[\frac{\Gamma^{n}\left(\frac{1}{2}\right)}{2^{n-1} \Gamma\left(\frac{n}{2}\right)}\right]^{2} C(\lambda) \frac{1}{\varepsilon}(1+o(1)), \varepsilon \rightarrow 0^{+} .
$$

Proof.

$$
\begin{aligned}
& A=\frac{\Gamma^{n}\left(\frac{1}{2}\right)}{2^{n} \Gamma\left(\frac{n}{2}\right)} \int_{\|x\| \geq 1}\|x\|^{-n-\frac{\varepsilon}{p}} \mathrm{~d} x \int_{\frac{1}{\|x\|}}^{\infty}\left(\frac{\min (1, u)}{1+u}\right)^{\lambda}\|x\|^{-\frac{\varepsilon}{q}} 2 u^{-1-\frac{\varepsilon}{q}} \mathrm{~d} u \\
& =\frac{\Gamma^{n}\left(\frac{1}{2}\right)}{2^{n-1} \Gamma\left(\frac{n}{2}\right)} \int_{\|x\| \geq 1}\|x\|^{-n-\varepsilon} \mathrm{d} x \int_{\frac{1}{\|x\|}}^{\infty}\left(\frac{\min (1, u)}{1+u}\right)^{\lambda} u^{-1-\frac{\varepsilon}{q}} \mathrm{~d} u \geq\left[\frac{\Gamma^{n}\left(\frac{1}{2}\right)}{2^{n-1} \Gamma\left(\frac{n}{2}\right)}\right]^{2} C(\lambda) \frac{1}{\varepsilon}(1+o(1)), \varepsilon \rightarrow 0^{+} .
\end{aligned}
$$




\section{Main results}

Theorem 1 If $p>1,1 / p+1 / q=1, n \in \square, 0<\lambda<1$, satisfy

$$
0<\int_{\square_{+}^{n}}\|x\|^{n(p-1)} f^{p}(x) \mathrm{d} x<\infty, 0<\int_{\square_{+}^{n}}\|y\|^{n(q-1)} g^{q}(y) \mathrm{d} y<\infty .
$$

Then

$$
J:=\int_{\square_{+}^{n}} \int_{\square_{+}^{n}}\left(\frac{\min \{\|x\|,\|y\|\}}{\|x\|+\|y\|}\right)^{\lambda} f(x) g(y) \mathrm{d} x \mathrm{~d} y<H_{n}(\lambda)\left[\int_{\eta_{+}}\|x\|^{n(p-1)} f^{p}(x) \mathrm{d} x\right]^{\frac{1}{p}}\left[\int_{{ }_{+}}\|y\|^{n(q-1)} g^{q}(y) \mathrm{d} y\right]^{\frac{1}{q}} ;
$$

where the constant factors $H_{n}(\lambda)$ is the best possible.

Proof. By H\&der's inequality, one has

$$
\begin{aligned}
& J=\int_{\square_{+}^{n}} \int_{\square_{+}^{n}}\left(\frac{\min \{\|x\|,\|y\|\}}{\|x\|+\|y\|}\right)^{\lambda} \frac{\|x\|^{\frac{n}{q}}}{\|y\|^{\frac{n}{p}}} f(x) \frac{\|y\|^{\frac{n}{p}}}{\|x\|^{\frac{n}{q}}} g(y) \mathrm{d} x \mathrm{~d} y \\
& \leq\left\{\int_{\square_{+}^{n}} \int_{\square_{+}^{n}}\left(\frac{\min \{\|x\|,\|y\|\}}{\|x\|+\|y\|}\right)^{\lambda} \frac{\|x\|^{n(p-1)}}{\|y\|^{n}} f^{p}(x) \mathrm{d} x \mathrm{~d} y\right\}^{\frac{1}{p}}\left\{\int_{\square_{+}^{n}} \int_{\square_{+}^{n}}\left(\frac{\min \{\|x\|,\|y\|\}}{\|x\|+\|y\|}\right)^{\lambda} \frac{\|y\|^{n(q-1)}}{\|x\|^{n}} g^{q}(y) \mathrm{d} x \mathrm{~d} y\right\}^{\frac{1}{q}} \\
& =\left[\int_{\square_{+}^{n}} w_{\lambda}(x)\|x\|^{n(p-1)} f^{p}(x) \mathrm{d} x\right]^{\frac{1}{p}}\left[\int_{\square_{+}^{n}} w_{\lambda}(y)\|y\|^{n(q-1)} g^{q}(y) \mathrm{d} y\right]^{\frac{1}{q}} .
\end{aligned}
$$

If this inequality takes the form an equality, which contradicts (5), hence we have

$$
J<\left[\int_{\square_{+}^{n}} w_{\lambda}(x)\|x\|^{n(p-1)} f^{p}(x) \mathrm{d} x\right]^{\frac{1}{p}}\left[\int_{\square_{+}^{n}} w_{\lambda}(y)\|y\|^{n(q-1)} g^{q}(y) \mathrm{d} y\right]^{\frac{1}{q}} . .
$$

By lemma 2, we have

$$
J<H_{n}(\lambda)\left[\int_{\square_{+}^{n}}\|x\|^{n(p-1)} f^{p}(x) \mathrm{d} x\right]^{\frac{1}{p}}\left[\int_{\square_{+}^{n}}\|y\|^{n(q-1)} g^{q}(y) \mathrm{d} y\right]^{\frac{1}{q}} \text {. Hence (6) is valid. }
$$

If the constant factor $H_{n}(\lambda)$ in (6) is not the best possible, then exists a positive number $k$ ( with $k<H_{n}(\lambda)$ ), such that(6) is still valid if one replaces $H_{n}(\lambda)$ by $k$. For $0<\varepsilon<\lambda /(2 q)$, sitting

$$
\begin{gathered}
f_{\varepsilon}(x)=\left\{\begin{array}{cc}
\|x\|^{-n-\frac{\varepsilon}{p}}, & \|x\| \geq 1 \\
0, & \|x\|<1,
\end{array} \quad g_{\varepsilon}(y)=\left\{\begin{array}{cl}
\|y\|^{-n-\frac{\varepsilon}{q}}, & \|y\| \geq 1 \\
0, & \|y\|<1,
\end{array}\right.\right. \\
\int_{\square_{+}^{n}+} \int_{\square_{+}^{n}}\left(\frac{\min \{\|x\|,\|y\|\}}{\|x\|+\|y\|}\right)^{\lambda} f_{\varepsilon}(x) g_{\varepsilon}(y) \mathrm{d} x \mathrm{~d} y<k\left[\int_{\eta+}\|x\|^{n(p-1)} f_{\varepsilon}^{p}(x) \mathrm{d} x\right]^{\frac{1}{p}}\left[\int_{{ }_{+}}\|y\|^{n(q-1)} g_{\varepsilon}^{q}(y) \mathrm{d} y\right]^{\frac{1}{q}},
\end{gathered}
$$

by lemma 3

$$
\begin{aligned}
& \int_{\square_{+}^{n}} \int_{\square_{+}^{n}}\left(\frac{\min \{\|x\|,\|y\|\}}{\|x\|+\|y\|}\right)^{\lambda} f_{\varepsilon}(x) g_{\varepsilon}(y) \mathrm{d} x \mathrm{~d} y \geq\left[\frac{\Gamma^{n}\left(\frac{1}{2}\right)}{2^{n-1} \Gamma\left(\frac{n}{2}\right)}\right]^{2} C(\lambda) \frac{1}{\varepsilon}(1+o(1)), \\
& {\left[\int_{\square_{+}^{n}}\|x\|^{n(p-1)} f_{\varepsilon}^{p}(x) \mathrm{d} x\right]^{\frac{1}{p}}\left[\int_{\square_{+}^{n}}\|y\|^{n(q-1)} g_{\varepsilon}^{q}(y) \mathrm{d} y\right]^{\frac{1}{q}}=\int_{\|x\| \geq 1}\|x\|^{-n-\varepsilon} d x=\frac{\Gamma^{n}\left(\frac{1}{2}\right)}{2^{n-1} \Gamma\left(\frac{n}{2}\right)} \cdot \frac{1}{\varepsilon} .}
\end{aligned}
$$


Hence, we have $\left[\frac{\Gamma^{n}\left(\frac{1}{2}\right)}{2^{n-1} \Gamma\left(\frac{n}{2}\right)}\right]^{2} C(\lambda) \frac{1}{\varepsilon}(1+o(1)) \leq k \cdot \frac{\Gamma^{n}\left(\frac{1}{2}\right)}{2^{n-1} \Gamma\left(\frac{n}{2}\right)} \cdot \frac{1}{\varepsilon}$.

For $\varepsilon \rightarrow 0^{+}$. we have $H_{n}(\lambda) \leq k$. This contradicts the fact that $k<H_{n}(\lambda)$. Hence the constant factor in $(6)$ is the best possible.

\section{References}

[1] M. Gao, B. Yang, Proceeding of the American Mathematical Society, vol. 126, no. 3, 751-759, 1998.

[2] G. H. Hardy, J. E. Littlewood and G. Pólya, Inequalities, Gambridge University Press, Gambridge, 1952.

[3] Y. Hong, Acta Mathematics Sinica, vol. 44, no.4, 619-626, 2001.(in chinese)

[4] Y. Hong, Journal of Inequalities in Pure and Applied Mathematics, vol. 7, no. 4, article 139, 2006.

[5] L. Hua, An Introduction to advanced mathematics ( Remaining sections), Science publishers, Beijing, 1984.

[6] J. Kuang, L. Debnath, Journal of Mathematical Analysis and Applications, vol.245, no.1, 248-265, 2000.

[7] J. Kuang, Applied Inequalities, Shandong Science and Technology Press, Jinan, 2004.

[8] D. S. Mitrinovi, J. E. Pecaric and A. M. Fink, Inequalities Involving Functions and Their Integrals and Derivatives, Kluwer Academic Publishers, Boston, 1991.

[9] B. G Pachpatte, Journal of Mathematical Analysis and Applications, vol.226, no.1, 166-179,1998.

[10] B. Yang and L. Debnath, On a new strengthened Hardy-Hilbert's inequality, International Journal of Mathematics and Mathematical Sciences, vol.21, 403-408.1998

[11] B. Yang, On a generalization of Hardy Hilbert's Integral inequality with a best value Chinese Annals of Mathematics, Series A, vol.21, 401-408, 2000.

[12] B. Yang, On a generalization of a Hilbert's Type Integral inequality and Its Applications Mathematica Applicata, vol.16, 82-86, 2003.

[13] B. Yang, On a multiple Hardy Hilbert's Integral inequality, Chinese Annals of Mathematics, Series A, vol.24, 743-750, 2003.

[14] B. Sun, A Multiple Hilbert type integral inequality with the best contant factor, Journal of inequalities and Applications, Article ID 71049, 14 pages, 2007.

[15] Z. Wang, D. Guo, Theory of particular function, Science press, Beijing. 